\title{
Coherent states for a quantum particle on a circle
}

\author{
K Kowalski†, J Rembieliński† and L C Papaloucasł \\ $\dagger$ Department of Theoretical Physics, University of Łódź, ul. Pomorska 149/153, \\ 90-236 Łódź, Poland \\ $\ddagger$ Department of Mathematics, University of Athens, Panepistomiopolis, 15784 \\ Athens, Greece
}

\begin{abstract}
The coherent states for the quantum particle on the circle are introduced. The Bargmann representation within the actual treatment provides the representation of the algebra $[\hat{J}, U]=U$, where $U$ is unitary, which is a direct consequence of the Heisenberg algebra $[\hat{\varphi}, \hat{J}]=i$, but it is more adequate for the study of the circlular motion.
\end{abstract}

Key words: quantum mechanics, coherent states, quantum groups

PACS numbers: $02.10,02.20,02.30,02.40,03.65$

Short title: Coherent states for a quantum particle on a circle

August 8, 2018 


\section{Introduction}

In spite of their importance the theory of quantization of systems involving periodic motion seems to be far from complete. For instance, the difficulties has been recently reported with quantization of the plane pendulum [1]. Namely, different results were obtained depending whether the constraints were imposed before or after the quantization. Another example is yet unsolved problem of quantization of the double pendulum [2]. We also recall the troublesome questions [3] arising in the analysis of the Heisenberg uncertainty relations arising in the case when one of observables, as for instance the angle operator, has the compact spectrum.

The experience with the harmonic oscillator suggests that the concept of coherent states would be an important tool for the better understanding of the periodic motion of a quantum particle. Furthermore, it is natural to restrict to the simplest case of the quantum particle moving in a circle.

The purpose of this work is to introduce the coherent states for the quantum particle on a circle. As a matter of fact there exists a method for generating coherent states for the quantum particle moving in a circle from the standard coherent states [4]. The obtained coherent states are different from those defined in this paper. Nevertheless, it seems to us that the approach presented herein is more adequate one.

In section 2 we introduce the algebra $[\hat{J}, U]=U$, where $\hat{J}$ is the angular momentum operator and $U$ is unitary. This algebra is implied by the Heisenberg algebra $[\hat{\varphi}, \hat{J}]=i$, where $\hat{\varphi}$ is the angle operator, but it seems to be more adequate in the case with the circular motion of a quantum particle. In section 3 we define the abstract coherent states and discuss their basic properties. Section 4 is devoted to the Bargmann representation within the actual treatment. This representation provides us with the concrete realization of the algebra $[\hat{J}, U]=U$, in the space of functions possessing Laurent series expansion. In section 5 we illustrate the actual treatment by an example of the free motion of the quantum particle in a circle.

\section{Quantum mechanics on the circle}

The purpose of this section is to discuss the fundamental commutation relations and abstract Hilbert space of states for the quantum particle on the circle. In order to clarify the notation we now specialize to the case of the free motion in a circle. The classical Lagrangian is given by

$$
L=\frac{1}{2} \dot{\varphi}^{2}
$$


where we have assumed that the particle has the unit mass and it moves in the unit circle. The angular momentum canonically conjugate to the angle $\varphi$ is

$$
J=\frac{\partial L}{\partial \dot{\varphi}}=\dot{\varphi},
$$

so that the Hamiltonian can be written as

$$
H=\frac{1}{2} J^{2} .
$$

Clearly, we have the Poissson bracket such that

$$
\{\varphi, J\}=1,
$$

which leads accordingly to the rules of the canonical quantization to the commutator

$$
[\hat{\varphi}, \hat{J}]=i,
$$

where we set $\hbar=1$. The commutator (2.5) needs very subtle analysis $[3,5,6]$ involving common domain of operators $\hat{\varphi}$ and $\hat{J}$. On the other hand, the experience shows that the study of the circular motion of the quantum particle based on the formula (2.5) is technically complicated. It seems to us that the reason of difficulties with the application of the commutator (2.5) is the inadequate choice of $\hat{\varphi}$ as the position observable for the quantum particle on a circle. Indeed, this observable arising from utilization of the usual position operator for the particle on the real line does not take into account the topology of the circle. In our opinion the good candidate to represent the position of the quantum particle on the (unit) circle is the unitary operator $U$ such that

$$
U=e^{i \hat{\varphi}} .
$$

In fact, the spectrum of $U$ should obviously coincide with the unit circle. It should also be noted that $U$ is determined on the whole Hilbert space of states. Now, taking into account (2.5) and (2.6) we arrive at the following algebra:

$$
[\hat{J}, U]=U,
$$

where $U$ is unitary. It is easy to verify with the help of (2.7) that the spectrum of $U$ really coincides with the unit circle.

Consider the eigenvalue equation

$$
\hat{J}|j\rangle=j|j\rangle .
$$

From (2.8) and (2.7) it follows that the operators $U$ and $U^{\dagger}$ act on the vectors $|j\rangle$ as the rising and lowering operators, respectively, i.e. we have

$$
\begin{aligned}
U|j\rangle & =|j+1\rangle, \\
U^{\dagger}|j\rangle & =|j-1\rangle .
\end{aligned}
$$

In view of (2.9) we can generate the whole basis $\{|j\rangle\}$ of the Hilbert space of states from the unique vector (vacuum vector) $\left|j_{0}\right\rangle$, where $j_{0} \in[0,1]$. Clearly, different $j_{0}$ lead to 
nonequivalent irreducible representations of the commutation relations (2.7). We now demand the time-reversal invariance of the algebra (2.7), that is

$$
\begin{aligned}
T \hat{J} T^{-1} & =-\hat{J}, \\
T U T^{-1} & =U^{-1},
\end{aligned}
$$

where $T$ is the anti-unitary operator of time inversion. The relations (2.8)-(2.11) taken together yield

$$
T|j\rangle=|-j\rangle \text {. }
$$

Hence, we find that $T$ is well-defined on the Hilbert space of states generated by the vectors $|j\rangle$ if and only if the spectrum of $\hat{J}$ is symmetric with respect to zero. By virtue of (2.9) this means that the only possibility left is $j_{0}=0$ or $j_{0}=\frac{1}{2}$. Evidently, $j_{0}=0\left(j_{0}=\frac{1}{2}\right)$ implies integer (half-integer) eigenvalues $j$. We have thus shown by demanding the invariance of (2.7) under the time inversions which seems to be reasonable assumption provided we do not take into consideration dissipative systems, that $j$ can be only integer or half-integer. Notice, that the most natural interpretation of the case with integer (half-integer) $j$ is that it refers to bosons (fermions). The fact that solutions to the Schrödinger equation for the quantum pendulum involve spin-half eigenfunctions was recognized by Pradhan et al [7]. We also remark that the subalgebra (2.7) of the full algebra (2.7), (2.10) and (2.11) was postulated for the study of quantum mechanics on the circle in [8]. Nevertheless, the physical interpretation of the infinite number of nonequivalent irreducible representations of (2.7) arising when one restricts to (2.7) was not provided therein. On the other hand, it seems that the quantization procedure based solely on (2.7) which does not preserve such the fundamental symmetry of the free motion of the classical particle in the circle as the time-reversal one is not physically acceptable. We finally write down the orthogonality and completeness conditions satisfied by the vectors $|j\rangle$ such that

$$
\begin{aligned}
\langle j \mid k\rangle & =\delta_{j k}, \\
\sum_{j=-\infty}^{\infty}|j\rangle\langle j| & =I
\end{aligned}
$$

(substitution $j \rightarrow j-\frac{1}{2}$ in the case with fermions understood).

\section{Abstract coherent states}

\subsection{Definition of coherent states}

In this section we introduce the abstract coherent states for the quantum mechanics on the circle. We begin with the definition of these states. Since the generators $U$ and $U^{\dagger}$ of the algebra (2.7) are non-Hermitian (they are unitary), therefore we cannot adopt the usual technique of Perelomov [9] for generating coherent states. Instead, we follow 
the Barut-Girardello approach [10] and we seek the coherent states as the solution of the eigenvalue equation

$$
X|\xi\rangle=\xi|\xi\rangle,
$$

with complex $\xi$. What is $X$ ? Motivated by the form of the eigenvalue equation satisfied by the standard coherent states $|z\rangle$, where $z$ is complex, such that

$$
e^{i a}|z\rangle=e^{i z}|z\rangle,
$$

where $a \sim \hat{q}+i \hat{p}$ is the standard Bose annihilation operator and $\hat{q}$ and $\hat{p}$ are the position and momentum observables, respectively, we put

$$
X:=e^{i(\hat{\phi}+i \hat{J})} .
$$

Hence, using (2.6) and the Baker-Hausdorff formula we find

$$
X=U e^{-\hat{J}-\frac{1}{2}} .
$$

A straightforward calculation shows that the operators $X$ and $X^{\dagger}$ are the ladder operators. Namely,

$$
\begin{aligned}
X|j\rangle & =e^{-j-\frac{1}{2}}|j+1\rangle, \\
X^{\dagger}|j\rangle & =e^{-j+\frac{1}{2}}|j-1\rangle .
\end{aligned}
$$

It should also be noted that the operator $X$ transforms under the time inversions as (see (3.4), (2.10) and (2.11)):

$$
T X T^{-1}=X^{-1} .
$$

Now, expanding the coherent state $|\xi\rangle$ in the basis $|j\rangle$

$$
|\xi\rangle=\sum_{j=-\infty}^{\infty} c_{j}(\xi)|j\rangle,
$$

where $c_{j}(\xi)=\langle j \mid \xi\rangle$, and taking into account (3.1) and (3.5a) we obtain the following recursive relation:

$$
c_{j+1}=\xi^{-1} e^{-j-\frac{1}{2}} c_{j},
$$

where $j$ is integer. Solving this elementary recurrence we get

$$
c_{j}=c_{0}(\xi) \xi^{-j} e^{-\frac{j^{2}}{2}},
$$

where $c_{0}(\xi)$ is an arbitrary function of $\xi$. For the sake of simplicity we shall set in the sequel $c_{0}(\xi) \equiv 1$. Therefore,

$$
\langle j \mid \xi\rangle=\xi^{-j} e^{-\frac{j^{2}}{2}},
$$


and the coherent state $|\xi\rangle$ is given by

$$
|\xi\rangle=\sum_{j=-\infty}^{\infty} \xi^{-j} e^{-\frac{j^{2}}{2}}|j\rangle .
$$

We note that in accordance with usual properties of coherent states the complex numbers $\xi$ should parametrize the cylinder which is the classical phase space for the particle moving in a circle. Thus we can set

$$
\xi=e^{-l+i \varphi} .
$$

Indeed, consider the cylinder

$$
x=\cos \varphi, \quad y=\sin \varphi, \quad z=l .
$$

In order to identify the points of the cylinder with the (compactified) complex plane one should to deform it and then project the points of the obtained surface on the $(x, y)$ plane. It seems that the simplest possibility left is to utilize the following transformation:

$$
x^{\prime}=e^{-z} x, \quad y^{\prime}=e^{-z} y, \quad z^{\prime}=z,
$$

which leads to the representation (3.12). The correspondence between the parameters $l, \varphi$ and the classical angular momentum and angle variables, respectively, is discussed in the next subsection. We observe that the singularity in (3.11) corresponding to $\xi=0$ arises by virtue of (3.12) only for asymptotical values of $l$. It should be noted that the operator $X^{-1}$ acts on the coherent states as follows:

$$
X^{-1}|\xi\rangle=\xi^{-1}|\xi\rangle .
$$

We also write down the relation describing transformation of the coherent states under the time inversions such that

$$
T|\xi\rangle=\left|\xi^{*-1}\right\rangle,
$$

where the asterisk designates the complex conjugation.

In view of (3.12) we can write the coherent state in the form

$$
|l, \varphi\rangle=\sum_{j=-\infty}^{\infty} e^{l j-i j \varphi} e^{-\frac{j^{2}}{2}}|j\rangle,
$$

where $|l, \varphi\rangle \equiv|\xi\rangle$ with $\xi=e^{-l+i \varphi}$. Evidently, we have

$$
\langle j \mid l, \varphi\rangle=e^{l j-i j \varphi} e^{-\frac{j^{2}}{2}} .
$$

We remark that the coherent states obey

$$
e^{-\eta \hat{J}}|\xi\rangle=\left|e^{\eta} \xi\right\rangle .
$$

Therefore, we can represent the state $|\xi\rangle$ as

$$
|\xi\rangle=e^{-(\ln \xi) \hat{J}}|1\rangle,
$$


where by virtue of $(3.11)$

$$
|1\rangle=\sum_{j=-\infty}^{\infty} e^{-\frac{j^{2}}{2}}|j\rangle .
$$

In the parametrization $l, \varphi$ the above formulas take the form

$$
e^{(h-i \psi) \hat{J}}|l, \varphi\rangle=|l+h, \varphi+\psi\rangle
$$

Hence,

$$
|l, \varphi\rangle=e^{(l-i \varphi) \hat{J}}|0,0\rangle
$$

where $|0,0\rangle \equiv|1\rangle$ (see (3.12) and (3.17)). The relations (3.19) and (3.22) follow directly from (2.8) and (3.11). Observe that the vector $|1\rangle$ is invariant under the action of the operator $T$ (see $(3.16))$.

The coherent states are not orthogonal. In fact, using (3.10) and (3.11) we find

$$
\begin{aligned}
& \langle\xi \mid \eta\rangle=\sum_{j=-\infty}^{\infty}\left(\xi^{*} \eta\right)^{-j} e^{-j^{2}}=\theta_{3}\left(\frac{i}{2 \pi} \ln \xi^{*} \eta \mid \frac{i}{\pi}\right), \quad \text { (boson case) } \\
& \langle\xi \mid \eta\rangle=\sum_{j=-\infty}^{\infty}\left(\xi^{*} \eta\right)^{-j+\frac{1}{2}} e^{-\left(j-\frac{1}{2}\right)^{2}}=\theta_{2}\left(\frac{i}{2 \pi} \ln \xi^{*} \eta \mid \frac{i}{\pi}\right), \quad \text { (fermion case). }
\end{aligned}
$$

Accordingly, in the parametrization (3.12) and (3.17)

$$
\begin{aligned}
& \langle l, \varphi \mid h, \psi\rangle=\theta_{3}\left(\frac{1}{2 \pi}(\varphi-\psi)-\frac{l+h}{2} \frac{i}{\pi} \mid \frac{i}{\pi}\right), \quad \text { (boson case) } \\
& \langle l, \varphi \mid h, \psi\rangle=\theta_{2}\left(\frac{1}{2 \pi}(\varphi-\psi)-\frac{l+h}{2} \frac{i}{\pi} \mid \frac{i}{\pi}\right) . \quad \text { (fermion case) }
\end{aligned}
$$

The functions $\theta_{3}$ and $\theta_{2}$ in the above formulas are the Jacobi theta-functions defined by

$$
\begin{aligned}
& \theta_{3}(v \mid \tau)=\sum_{n=-\infty}^{\infty} q^{n^{2}}\left(e^{i \pi v}\right)^{2 n} \\
& \theta_{2}(v \mid \tau)=\sum_{n=-\infty}^{\infty} q^{\left(n-\frac{1}{2}\right)^{2}}\left(e^{i \pi v}\right)^{2 n-1}
\end{aligned}
$$

where $q=e^{i \pi \tau}$ and $\operatorname{Im} \tau>0$. We note that

$$
\theta_{3}(-v)=\theta_{3}(v), \quad \theta_{2}(-v)=\theta_{2}(v) .
$$

The introduced coherent states are not normalized. Indeed, it follows immediately from (3.24) and (3.25) that

$$
\begin{aligned}
& \langle\xi \mid \xi\rangle=\theta_{3}\left(\frac{i}{\pi} \ln |\xi| \mid \frac{i}{\pi}\right), \quad \text { (boson case), } \\
& \langle\xi \mid \xi\rangle=\theta_{2}\left(\frac{i}{\pi} \ln |\xi| \mid \frac{i}{\pi}\right), \quad \text { (fermion case) }
\end{aligned}
$$

and

$$
\begin{aligned}
& \langle l, \varphi \mid l, \varphi\rangle=\sum_{j=-\infty}^{\infty} e^{2 l j} e^{-j^{2}}=\theta_{3}\left(\frac{i l}{\pi} \mid \frac{i}{\pi}\right), \quad \text { (boson case) } \\
& \langle l, \varphi \mid l, \varphi\rangle=\sum_{j=-\infty}^{\infty} e^{2 l\left(j-\frac{1}{2}\right)} e^{-\left(j-\frac{1}{2}\right)^{2}}=\theta_{2}\left(\frac{i l}{\pi} \mid \frac{i}{\pi}\right) . \quad \text { (fermion case) }
\end{aligned}
$$


Let finally recall that nonorthogonality is one of the characteristic features of coherent states.

\subsection{Coherent states and the classical phase space}

As we have promised in the previous section we now discuss the parametrization (3.12) in a more detail. Consider the expectation value of the angular momentum operator $\hat{J}$ in the normalized coherent state. On using (2.8), (3.17), (3.18) and (3.29a) we find in the boson case

$$
\frac{\langle\xi|\hat{J}| \xi\rangle}{\langle\xi \mid \xi\rangle} \equiv \frac{\langle l, \varphi|\hat{J}| l, \varphi\rangle}{\langle l, \varphi \mid l, \varphi\rangle}=\frac{1}{2 \theta_{3}\left(\frac{i l}{\pi} \mid \frac{i}{\pi}\right)} \frac{d}{d l} \theta_{3}\left(\frac{i l}{\pi} \mid \frac{i}{\pi}\right) .
$$

Hence, taking into account the identity

$$
\theta_{3}\left(\frac{i l}{\pi} \mid \frac{i}{\pi}\right)=\sqrt{\pi} e^{l^{2}} \theta_{3}(l \mid i \pi)
$$

which follows directly from the general formula

$$
\theta_{3}\left(\frac{v}{\tau} \mid-\frac{1}{\tau}\right)=\sqrt{\frac{\tau}{i}} e^{\frac{i \pi v^{2}}{\tau}} \theta_{3}(v \mid \tau)
$$

we arrive at the following relation:

$$
\frac{\langle\xi|\hat{J}| \xi\rangle}{\langle\xi \mid \xi\rangle}=l+\frac{1}{2 \theta_{3}(l \mid i \pi)} \frac{d}{d l} \theta_{3}(l \mid i \pi)
$$

Now, using the identity [11]

$$
\frac{d \theta_{3}(v)}{d v}=\pi \theta_{3}(v)\left(\sum_{n=1}^{\infty} \frac{2 i q^{2 n-1} e^{2 i \pi v}}{1+q^{2 n-1} e^{2 i \pi v}}-\sum_{n=1}^{\infty} \frac{2 i q^{2 n-1} e^{-2 i \pi v}}{1+q^{2 n-1} e^{-2 i \pi v}}\right)
$$

we finally obtain the following equation:

$$
\frac{\langle\xi|\hat{J}| \xi\rangle}{\langle\xi \mid \xi\rangle}=l-2 \pi \sin (2 l \pi) \sum_{n=1}^{\infty} \frac{e^{-\pi^{2}(2 n-1)}}{\left(1+e^{-\pi^{2}(2 n-1)} e^{2 i l \pi}\right)\left(1+e^{-\pi^{2}(2 n-1)} e^{-2 i l \pi}\right)} .
$$

We note that for $l$ integer or half-integer the above formula reduces to

$$
\frac{\langle\xi|\hat{J}| \xi\rangle}{\langle\xi \mid \xi\rangle}=l
$$

that is $l$ is precisely the classical angular momentum. Otherwise, we observe that the very good approximation of (3.35) is

$$
\frac{\langle\xi|\hat{J}| \xi\rangle}{\langle\xi \mid \xi\rangle} \approx l-2 \pi e^{-\pi^{2}} \sin (2 l \pi) . \quad \text { (boson case) }
$$

Moreover, it can be easily calculated that the second term from the right-hand side of (3.37) is still negligible compared with $l$ (the quantum corrections are really small). The maximal error arising in the case $l \rightarrow 0$ is of order 0.1 per cent. Therefore, in any case

$$
\frac{\langle\xi|\hat{J}| \xi\rangle}{\langle\xi \mid \xi\rangle} \approx l \text {. }
$$


We have thus shown that the parameter $l$ in (3.12) can be identified (in general approximately) with the classical angular momentum. In order to demonstrate that the same holds true in the case with fermions we write down the identity

$$
\theta_{2}\left(\frac{i l}{\pi} \mid \frac{i}{\pi}\right)=\sqrt{\pi} e^{l^{2}} \theta_{4}(l \mid i \pi)
$$

where the Jacobi theta-function $\theta_{4}$ is defined as

$$
\theta_{4}(v)=\sum_{n=-\infty}^{\infty}(-1)^{n} q^{n^{2}}\left(e^{i \pi v}\right)^{2 n}
$$

and (3.39) is an immediate consequence of the general relation

$$
\theta_{2}\left(\frac{v}{\tau} \mid-\frac{1}{\tau}\right)=\sqrt{\frac{\tau}{i}} e^{\frac{i \pi v^{2}}{\tau}} \theta_{4}(v \mid \tau)
$$

By (3.39) we can reduce the equation

$$
\frac{\langle\xi|\hat{J}| \xi\rangle}{\langle\xi \mid \xi\rangle} \equiv \frac{\langle l, \varphi|\hat{J}| l, \varphi\rangle}{\langle l, \varphi \mid l, \varphi\rangle}=\frac{1}{2 \theta_{2}\left(\frac{i l}{\pi} \mid \frac{i}{\pi}\right)} \frac{d}{d l} \theta_{2}\left(\frac{i l}{\pi} \mid \frac{i}{\pi}\right)
$$

following from $(2.8)$, (3.18) with $j$ half-integer, and (3.29b), to

$$
\frac{\langle\xi|\hat{J}| \xi\rangle}{\langle\xi \mid \xi\rangle}=l+\frac{1}{2 \theta_{4}(l \mid i \pi)} \frac{d}{d l} \theta_{4}(l \mid i \pi) .
$$

Now, taking into account the identity

$$
\frac{d \theta_{4}(v)}{d v}=\pi \theta_{4}(v)\left(\sum_{n=1}^{\infty} \frac{2 i q^{2 n-1} e^{-2 i \pi v}}{1-q^{2 n-1} e^{-2 i \pi v}}-\sum_{n=1}^{\infty} \frac{2 i q^{2 n-1} e^{2 i \pi v}}{1-q^{2 n-1} e^{2 i \pi v}}\right),
$$

we finally arrive at the following relation:

$$
\frac{\langle\xi|\hat{J}| \xi\rangle}{\langle\xi \mid \xi\rangle}=l+2 \pi \sin (2 l \pi) \sum_{n=1}^{\infty} \frac{e^{-\pi^{2}(2 n-1)}}{\left(1-e^{-\pi^{2}(2 n-1)} e^{2 i l \pi}\right)\left(1-e^{-\pi^{2}(2 n-1)} e^{-2 i l \pi}\right)} .
$$

As with the case of bosons we find that for $l$ integer or half-integer the exact formula is valid such that

$$
\frac{\langle\xi|\hat{J}| \xi\rangle}{\langle\xi \mid \xi\rangle}=l
$$

Accordingly, for general $l$ we find that the very good approximation of (3.45) is given by

$$
\frac{\langle\xi|\hat{J}| \xi\rangle}{\langle\xi \mid \xi\rangle} \approx l+2 \pi e^{-\pi^{2}} \sin (2 l \pi) . \quad \text { (fermion case) }
$$

Proceeding as with (3.37) one can easily check that the approximation (3.47) is as good as

$$
\frac{\langle\xi|\hat{J}| \xi\rangle}{\langle\xi \mid \xi\rangle} \approx l
$$


It thus appears that analogously as with bosons in the case of fermions we can also identify the parameter $l$ with the classical angular momentum.

We now examine the role of the parameter $\varphi$ in (3.12). Consider first the case with bosons. Equations (3.17), (2.9a), (3.18) and (3.29) taken together yield

$$
\frac{\langle\xi|U| \xi\rangle}{\langle\xi \mid \xi\rangle} \equiv \frac{\langle l, \varphi|U| l, \varphi\rangle}{\langle l, \varphi \mid l, \varphi\rangle}=e^{-\frac{1}{4}} e^{i \varphi} \frac{\theta_{2}\left(\frac{i l}{\pi} \mid \frac{i}{\pi}\right)}{\theta_{3}\left(\frac{i l}{\pi} \mid \frac{i}{\pi}\right)} . \quad \text { (boson case) }
$$

Hence, making use of the relation

$$
\theta_{2}(v)=e^{i \pi\left(\frac{\tau}{4}+v\right)} \theta_{3}\left(v+\frac{\tau}{2}\right)
$$

and the identity (3.31) we obtain

$$
\frac{\langle\xi|U| \xi\rangle}{\langle\xi \mid \xi\rangle}=e^{-\frac{1}{4}} e^{i \varphi} \frac{\theta_{3}\left(l+\frac{1}{2} \mid i \pi\right)}{\theta_{3}(l \mid i \pi)} . \quad \text { (boson case) }
$$

Finally, taking into account the definition of the function $\theta_{3}$ given by (3.26a) we arrive at the following formula:

$$
\frac{\langle\xi|U| \xi\rangle}{\langle\xi \mid \xi\rangle}=e^{-\frac{1}{4}} e^{i \varphi} \frac{\sum_{j=-\infty}^{\infty} e^{-\pi^{2} j^{2}} e^{i \pi(2 l+1) j}}{\sum_{j=-\infty}^{\infty} e^{-\pi^{2} j^{2}} e^{i \pi 2 l j}} .
$$

A look at (3.52) is enough to conclude that the very good approximation of (3.52) is

$$
\frac{\langle\xi|U| \xi\rangle}{\langle\xi \mid \xi\rangle} \approx e^{-\frac{1}{4}} e^{i \varphi}
$$

We observe that the approximation (3.53) is valid also in the case of fermions. Indeed, using (3.17), (2.9a), (3.18) and (3.29) we get

$$
\frac{\langle\xi|U| \xi\rangle}{\langle\xi \mid \xi\rangle}=e^{-\frac{1}{4}} e^{i \varphi} \frac{\theta_{3}\left(\frac{i l}{\pi} \mid \frac{i}{\pi}\right)}{\theta_{2}\left(\frac{i l}{\pi} \mid \frac{i}{\pi}\right)} . \quad \text { (fermion case) }
$$

Comparing equation (3.54) with (3.49) we find that the approximation (3.53) holds true.

We now discuss (3.53) in a more detail. Due to the term $e^{-\frac{1}{4}}$ in (3.53), it turns out that the average value of $U$ in the normalized coherent state does not belong to the unit circle. In our opinion the reason of such discrepancy is the inadequate definition of the expectation value as the diagonal matrix element of $U$. The fact that average values in some states cannot be well-defined even in the case of Hermitian operators (observables) is known in the literature. We only recall the expectation value of the usual position operator for the particle on the real line in its eigenstate. Following approach analogous to that described in ref. 12 we introduce the relative average value of $U$ such that

$$
\frac{\langle U\rangle_{\xi}}{\langle U\rangle_{\eta}}:=\frac{\langle\xi|U| \xi\rangle}{\langle\eta|U| \eta\rangle}
$$


where $|\xi\rangle$ and $|\eta\rangle$ are the normalized coherent states. We stress that $\langle U\rangle_{\xi} \neq\langle\xi|U| \xi\rangle$. By virtue of (3.53) (see also (3.52)) we have

$$
\frac{\langle U\rangle_{\xi}}{\langle U\rangle_{\eta}} \approx e^{i(\varphi-\psi)},
$$

where $\xi=e^{-l+i \varphi}$ and $\eta=e^{-h+i \psi}$. In particular, we find (see (3.21))

$$
\frac{\langle U\rangle_{\xi}}{\langle U\rangle_{1}} \approx e^{i \varphi} .
$$

The form of (3.57) suggests that the relative expectation value $\langle U\rangle_{\xi} /\langle U\rangle_{1}$ is the most natural one to describe the average position of a quantum particle on the circle.

We have thus shown that the parameter $\varphi$ can be regarded as the classical angle. Notice that in general we have the approximate formulas (3.38) and (3.57). In our opinion, due to some immanent feature of the quantum mechanics on the circle, the approximation of the classical phase space expressed by (3.38) and (3.57) is the best possible one and in this sense the coherent states marked with points of such slightly deformed phase space are closest to the classical ones.

We end this section with discussion of the time evolution of expectation values of operators $\hat{J}, U$ and $X$ in the coherent state. We confine ourselves to the free motion in the circle. Evidently, the Hamiltonian is given by (see (2.3))

$$
\hat{H}=\frac{1}{2} \hat{J}^{2} .
$$

Taking into account (3.58) we get

$$
\hat{J}(t)=\hat{J},
$$

that is $\hat{J}$ is the integral of motion. Therefore, by virtue of (3.38)

$$
\frac{\langle\xi|\hat{J}(t)| \xi\rangle}{\langle\xi \mid \xi\rangle} \approx l .
$$

Thus, as expected, the angular momentum $l$ labelling the coherent state $|\xi\rangle$ is conserved during the time evolution. Furthermore, using the identity which follows immediately from $(2.7)$

$$
f(\hat{J}) U=U f(\hat{J}+1),
$$

where $f$ is an arbitrary analytic function, we find

$$
U(t)=e^{i t \frac{\hat{J}^{2}}{2}} U e^{-i t \frac{\hat{J}^{2}}{2}}=U e^{i t\left(\hat{J}+\frac{1}{2}\right)} .
$$

Now, we have the approximate formula (see appendix)

$$
\frac{\left\langle\xi\left|e^{s \hat{J}}\right| \xi\right\rangle}{\langle\xi \mid \xi\rangle} \approx e^{\frac{1}{4} s^{2}+s l},
$$


and the relation

$$
U^{\dagger}|\xi\rangle=\xi^{-1} e^{-\hat{J}-\frac{1}{2}}|\xi\rangle .
$$

The equation (3.64) is implied by (3.4) and

$$
X^{\dagger}|\xi\rangle=\xi^{-1} e^{-2 \hat{J}-1}|\xi\rangle
$$

which is a direct consequence of the identity (see (3.4))

$$
X^{\dagger} X=e^{-2 \hat{J}-1}
$$

and (3.15). Eqs. (3.62)-(3.64) taken together yield

$$
\frac{\langle\xi|U(t)| \xi\rangle}{\langle\xi \mid \xi\rangle} \approx e^{-\frac{t^{2}}{4}} e^{-\frac{1}{4}} e^{i(\varphi+t l)}
$$

Reasoning as with (3.53) we introduce the relative expectation value of $U(t)$ of the form

$$
\frac{\langle U(t)\rangle_{\xi}}{\langle U(t)\rangle_{\eta}}:=\frac{\langle\xi|U(t)| \xi\rangle}{\langle\eta|U(t)| \eta\rangle},
$$

where $|\xi\rangle$ and $|\eta\rangle$ are normalized coherent states, which in view of (3.67) leads to (see also (3.57))

$$
\frac{\langle U(t)\rangle_{\xi}}{\langle U(t)\rangle_{1}} \approx e^{i(\varphi+t l)}
$$

Notice that the phase in the exponential function from the right-hand side of (3.69) is nothing but the solution of the classical equations of the free motion in the circle such that

$$
\begin{aligned}
\dot{\varphi} & =l, \\
\dot{l} & =0 .
\end{aligned}
$$

We now discuss the average value of $X(t)$. Making use of (3.4) and (3.62) we obtain

$$
X(t)=e^{i t\left(\hat{J}-\frac{1}{2}\right)} X
$$

Hence, taking into account (3.1) and (3.63) we get

$$
\frac{\langle\xi|X(t)| \xi\rangle}{\langle\xi \mid \xi\rangle} \approx e^{-\frac{t^{2}}{4}} e^{-l+i\left(\varphi+t\left(l-\frac{1}{2}\right)\right)} .
$$

It follows immediately from (3.72) that the corresponding relative expectation value is

$$
\frac{\langle X(t)\rangle_{\xi}}{\langle X(t)\rangle_{1}} \approx e^{-l+i\left(\varphi+t\left(l-\frac{1}{2}\right)\right)} .
$$

On making use of the identification (see (3.12))

$$
\xi(t)=e^{-l(t)+i \varphi(t)}
$$


we find that the argument of the exponential function from (3.73) refers to the solution of the system

$$
\begin{aligned}
\dot{\varphi} & =l-\frac{1}{2}, \\
i & =0 .
\end{aligned}
$$

Evidently, (3.75) reduces to (3.70) in the semiclassical limit $|l| \gg 1$.

We finally point out that the coherent states are not stable with respect to the free evolution (the standard coherent states are clearly also unstable in the case with the free evolution - recall spreading of wave packets). Indeed, using (3.71), (2.8), (3.1) and (3.17) we arrive at the following formula:

$$
X(t)|l, \varphi\rangle=e^{-l+i\left(\varphi-\frac{t}{2}\right)}|l, \varphi-t\rangle .
$$

The natural dynamics for the coherent states which ensures their stability is that described by the Hamiltonian of the form

$$
\hat{H}=\omega \hat{J} .
$$

In fact, we have

$$
U(t)=e^{i t \omega \hat{J}} U e^{-i t \omega \hat{J}}=e^{i t \omega} U .
$$

Hence, by virtue of (3.4)

$$
X(t)=e^{i t \omega} X
$$

which leads to

$$
X(t)|\xi\rangle=\xi(t)|\xi\rangle
$$

where $\xi(t)=e^{i t \omega} \xi$.

\subsection{Minimalization of the Heisenberg uncertainty relations}

In this section we show that the introduced coherent states minimalize the Heisenberg uncertainty relations. Let us define the following Hermitian operators:

$$
\begin{aligned}
Q & :=\frac{1}{2}\left(X+X^{\dagger}\right), \\
P & :=\frac{1}{2 i}\left(X-X^{\dagger}\right),
\end{aligned}
$$

where $X$ is given by (3.4). Notice that the operators $Q$ and $P$ play the same role as the usual position and momentum operators in the case with the standard coherent states. Consider the Heisenberg uncertainty relation

$$
\Delta_{\phi} Q \Delta_{\phi} P \geq \frac{1}{2} \frac{\langle\phi|[Q, P]| \phi\rangle}{\langle\phi \mid \phi\rangle} .
$$


Taking into account (3.81) and the identity

$$
X X^{\dagger}=e^{2} X^{\dagger} X
$$

where $e=\exp (1)$, which can be derived easily by means of (3.66), (3.4) and (3.61), we obtain

$$
[Q, P]=-\frac{1}{2 i}\left(e^{2}-1\right) X^{\dagger} X
$$

By virtue of (3.84) and (3.1) we have

$$
\frac{\langle\xi|[Q, P]| \xi\rangle}{\langle\xi \mid \xi\rangle}=-\frac{1}{2 i}\left(e^{2}-1\right) \xi^{*} \xi=-\frac{1}{2 i}\left(e^{2}-1\right) e^{-2 l} .
$$

Now, equations (3.81), (3.83) and (3.12) taken together yield

$$
\begin{aligned}
\frac{\langle\xi|Q| \xi\rangle}{\langle\xi \mid \xi\rangle} & =\frac{1}{2}\left(\xi+\xi^{*}\right)=e^{-l} \cos \varphi \\
\frac{\langle\xi|P| \xi\rangle}{\langle\xi \mid \xi\rangle} & =\frac{1}{2 i}\left(\xi-\xi^{*}\right)=e^{-l} \sin \varphi \\
\frac{\left\langle\xi\left|Q^{2}\right| \xi\right\rangle}{\langle\xi \mid \xi\rangle} & =\frac{1}{4}\left[\left(\xi+\xi^{*}\right)^{2}+\left(e^{2}-1\right) \xi^{*} \xi\right]=\frac{1}{4} e^{-2 l}\left[2 \cos 2 \varphi+e^{2}+1\right] \\
\frac{\left\langle\xi\left|P^{2}\right| \xi\right\rangle}{\langle\xi \mid \xi\rangle} & =-\frac{1}{4}\left[\left(\xi-\xi^{*}\right)^{2}-\left(e^{2}-1\right) \xi^{*} \xi\right]=-\frac{1}{4} e^{-2 l}\left[2 \cos 2 \varphi-\left(e^{2}+1\right)\right]
\end{aligned}
$$

As a consequence of (3.85) and (3.86) we find

$$
\Delta_{\xi} Q \Delta_{\xi} P=\frac{1}{2} \frac{\langle\xi|[Q, P]| \xi\rangle}{\langle\xi \mid \xi\rangle}
$$

That is the (normalized) coherent states minimalize the Heisenberg uncertainty relation (3.82). We point out that such minimalization does not take place in the case of the vectors $|j\rangle$ (see (2.8)). Indeed, a quick calculation based on (3.5) and the identity (3.66) shows that this is not the case.

\section{Bargmann representation}

\subsection{Inner product}

This section is devoted to the Bargmann representation within the introduced formalism. We now return to eq. (3.18). Taking into account (3.18) and (2.13) we find that the resolution of the identity for the coherent states can be written as

$$
\frac{1}{2 \pi^{\frac{3}{2}}} \int_{0}^{2 \pi} d \varphi \int_{-\infty}^{\infty} d l e^{-l^{2}}|l, \varphi\rangle\langle l, \varphi|=I .
$$


On using the complex variable $\xi$ (see (3.12)) the completeness condition (4.1) takes the form

$$
\frac{1}{4 i \pi^{\frac{3}{2}}} \int_{\mathbf{C}} d \xi d \xi^{*} \frac{e^{-(\ln |\xi|)^{2}}}{|\xi|^{2}}|\xi\rangle\langle\xi|=I,
$$

where $\mathbf{C}$ is the complex plane. We point out that there is no singularity in (4.2) for $\xi=0$. Hence, we arrive at the functional representation such that

$$
\langle\phi \mid \psi\rangle=\frac{1}{4 i \pi^{\frac{3}{2}}} \int_{\mathbf{C}} d \xi d \xi^{*} \frac{e^{-(\ln |\xi|)^{2}}}{|\xi|^{2}}\left(\phi\left(\xi^{*}\right)\right)^{*} \psi\left(\xi^{*}\right),
$$

where $\phi\left(\xi^{*}\right)=\langle\xi \mid \phi\rangle$. The representation (4.3) is the counterpart of the Bargmann representation [13] applied in the theory of standard coherent states. The operators act in the representation (4.3) as follows:

$$
\begin{aligned}
\hat{J} \phi\left(\xi^{*}\right) & =-\xi^{*} \frac{d}{d \xi^{*}} \phi\left(\xi^{*}\right), \\
U \phi\left(\xi^{*}\right) & =\frac{\phi\left(e \xi^{*}\right)}{\sqrt{e} \xi^{*}}, \\
U^{\dagger} \phi\left(\xi^{*}\right) & =\frac{\xi^{*}}{\sqrt{e}} \phi\left(e^{-1} \xi^{*}\right), \\
T \phi\left(\xi^{*}\right) & =\left(\phi\left(\xi^{-1}\right)\right)^{*}, \\
X \phi\left(\xi^{*}\right) & =\frac{\phi\left(e^{2} \xi^{*}\right)}{e \xi^{*}}, \\
X^{\dagger} \phi\left(\xi^{*}\right) & =\xi^{*} \phi\left(\xi^{*}\right),
\end{aligned}
$$

where $e=\exp (1)$. In fact the relation (4.4) follows directly from (3.11) and (3.12). In order to derive (4.5), (4.6) and (4.8) we have used the formulas (3.64) and (3.65) as well as the identities

$$
\begin{aligned}
e^{\lambda \xi^{*} \frac{d}{d \xi^{*}}} & =\sum_{n=0}^{\infty} \frac{\left(e^{\lambda}-1\right)^{n}}{n !} \xi^{* n} \frac{d^{n}}{d \xi^{* n}}, \\
\phi\left(\lambda \xi^{*}\right) & =\sum_{n=0}^{\infty} \frac{(\lambda-1)^{n}}{n !} \xi^{* n} \frac{d^{n}}{d \xi^{* n}} \phi\left(\xi^{*}\right),
\end{aligned}
$$

implying

$$
\phi\left(e^{\lambda} \xi^{*}\right)=e^{\lambda \xi^{*} \frac{d}{d \xi^{*}}} \phi\left(\xi^{*}\right) .
$$

We remark that the relation (4.10) is well-known in the boson calculus [14]. Finally, the equations (4.7) and (4.9) are immediate consequences of (3.1) and (3.16), respectively.

What are the elements $\phi\left(\xi^{*}\right)$ of the Hilbert space specified by (4.3) ? In view of (3.10) the basis vectors $|j\rangle$ are represented by the functions

$$
e_{j}\left(\xi^{*}\right) \equiv\langle\xi \mid j\rangle=e^{-\frac{j^{2}}{2}} \xi^{*-j} .
$$


Using (4.5) and (4.6) it can be checked easily that

$$
\begin{aligned}
U e_{j}\left(\xi^{*}\right) & =e_{j+1}\left(\xi^{*}\right), \\
U^{\dagger} e_{j}\left(\xi^{*}\right) & =e_{j-1}\left(\xi^{*}\right) .
\end{aligned}
$$

Reasoning as in section 2 we arrive at the representation generated by means of (4.14) from the vacuum vector

$$
e_{0}\left(\xi^{*}\right) \equiv 1
$$

in the case with bosons, and the representation for fermions with the vacuum vector given by

$$
e_{\frac{1}{2}}\left(\xi^{*}\right)=e^{-\frac{1}{4}} \xi^{*-\frac{1}{2}}
$$

Clearly, the space of the irreducible representation is the Hilbert space with the inner product (4.3) of functions possessing the Laurent series expansion such that

$$
\phi\left(\xi^{*}\right)=\sum_{j=-\infty}^{\infty} c_{j} \xi^{*-j}, \quad \text { (boson case) }
$$

in the case of bosons, and the space of functions of the form

$$
\phi\left(\xi^{*}\right)=\sum_{j=-\infty}^{\infty} c_{j} \xi^{*-j+\frac{1}{2}}, \quad \text { (fermion case) }
$$

in the case with fermions.

Observe that the expressions from the right-hand side of (4.5), (4.6) and (4.8) have the structure similar to $q$-derivatives in the theory of quantum groups. Such resemblance is not an accidental one. Indeed, return to the equation (3.83). The formula (3.83) shows that $X$ and $X^{\dagger}$ are nothing but the generators of the complex Manin's plane with involution [15]. On the other hand, we have the commutation relations

$$
\begin{aligned}
& {\left[X, X^{\dagger}\right]=2 \sinh 1 e^{-2 \hat{J}},} \\
& {[\hat{J}, X]=X, \quad\left[\hat{J}, X^{\dagger}\right]=-X^{\dagger} .}
\end{aligned}
$$

Hence, introducing the operator $N$ of the form

$$
N:=-\hat{J}+\frac{1}{2} \ln (2 \sinh 1)
$$

we arrive at the following algebra:

$$
\begin{aligned}
& {\left[X, X^{\dagger}\right]=\left(e^{2}\right)^{N},} \\
& {[N, X]=-X, \quad\left[N, X^{\dagger}\right]=X^{\dagger} .}
\end{aligned}
$$

Thus, it turns out that we deal with deformed Heisenberg algebra. Notice that the role of the deformation parameter $q$ is played in both formulas (3.83) and (4.21) by $e^{2}$. 
Therefore, the actual treatment provides us with the concrete value of $q$. As pointed out by a referee, on defining

$$
a=(1+q)^{-\frac{1}{2}} X, \quad a^{\dagger}=(1+q)^{-\frac{1}{2}} X^{\dagger},
$$

we can write (4.21) in the form of the usual $q$-boson algebra. Indeed, using (4.21) and (3.83) we find

$$
a a^{\dagger}-q a^{\dagger} a=q^{-N}, \quad[N, a]=-a, \quad\left[N, a^{\dagger}\right]=a^{\dagger},
$$

where $q=e^{-2}$. The representations of the algebra (4.23) were investigated in [16]. The formulas obtained therein in the case of $q<1$ can be regarded as the counterparts of the relations (2.8), (3.5) and (3.83) with $X$ expressed with the help of (4.22) by $a$, and $\hat{J}$ given by (4.20). Nevertheless, the physical interpretation of the representations of (4.23) in the context of the quantum mechanics on the circle was not discussed in [16]. The coherent states for the algebra (4.23) were studied in [17]. Nevertheless, it was assumed therein that as with standard coherent states the eigenvalues of the number operator $N$ are nonnegative integers. On the contrary, the eigenvalues of the operator $N$ given by (4.20) can be also negative and non-integer. We conclude that the coherent states discussed in [17] are different from those studied in this work.

\subsection{Reproducing kernel}

Recall that the existence of the reproducing kernel is one of the most characteristic properties of the standard Bargmann representation and its numerous generalizations. From (4.2) and (3.24) it follows immediately that the reproducing property can be written in the form

$$
\phi\left(\eta^{*}\right)=\frac{1}{4 i \pi^{\frac{3}{2}}} \int_{\mathbf{C}} d \xi d \xi^{*} \frac{e^{-(\ln |\xi|)^{2}}}{|\xi|^{2}} \mathcal{K}_{0, \frac{1}{2}}\left(\eta^{*}, \xi\right) \phi\left(\xi^{*}\right),
$$

where $\phi\left(\eta^{*}\right)=\langle\eta \mid \phi\rangle$ and the indices 0 and $\frac{1}{2}$ correspond to the case with bosons and fermions, respectively. The reproducing kernels $\mathcal{K}_{0, \frac{1}{2}}\left(\eta^{*}, \xi\right)$ are given by

$$
\begin{aligned}
& \mathcal{K}_{0}\left(\eta^{*}, \xi\right)=\theta_{3}\left(\frac{i}{2 \pi} \ln \eta^{*} \xi \mid \frac{i}{\pi}\right), \quad \text { (boson case) } \\
& \mathcal{K}_{\frac{1}{2}}\left(\eta^{*}, \xi\right)=\theta_{2}\left(\frac{i}{2 \pi} \ln \eta^{*} \xi \mid \frac{i}{\pi}\right) . \quad \text { (fermion case) }
\end{aligned}
$$

In particular, using (4.2) and (3.24) we get the following identities satisfied by the theta-functions $\theta_{3}$ and $\theta_{2}$ :

$$
\begin{aligned}
& \theta_{3}\left(\frac{i}{2 \pi} \ln \xi^{*} \eta \mid \frac{i}{\pi}\right)=\frac{1}{4 i \pi^{\frac{3}{2}}} \int_{\mathbf{C}} d \zeta d \zeta^{*} \frac{e^{-(\ln |\zeta|)^{2}}}{|\zeta|^{2}} \theta_{3}\left(\frac{i}{2 \pi} \ln \xi^{*} \zeta \mid \frac{i}{\pi}\right) \theta_{3}\left(\frac{i}{2 \pi} \ln \zeta^{*} \eta \mid \frac{i}{\pi}\right), \\
& \theta_{2}\left(\frac{i}{2 \pi} \ln \xi^{*} \eta \mid \frac{i}{\pi}\right)=\frac{1}{4 i \pi^{\frac{3}{2}}} \int_{\mathbf{C}} d \zeta d \zeta^{*} \frac{e^{-(\ln |\zeta|)^{2}}}{|\zeta|^{2}} \theta_{2}\left(\frac{i}{2 \pi} \ln \xi^{*} \zeta \mid \frac{i}{\pi}\right) \theta_{2}\left(\frac{i}{2 \pi} \ln \zeta^{*} \eta \mid \frac{i}{\pi}\right) .
\end{aligned}
$$


Taking into account (4.1) and (3.12) we arrive at the equivalent form of (4.26) such that

$$
\begin{aligned}
& \theta_{3}\left(\frac{1}{2 \pi}(\varphi-\psi)-\frac{l+h}{2} \frac{i}{\pi} \mid \frac{i}{\pi}\right) \\
& \quad=\frac{1}{2 \pi^{\frac{3}{2}}} \int_{0}^{2 \pi} d \alpha \int_{-\infty}^{\infty} d p e^{-p^{2}} \theta_{3}\left(\frac{1}{2 \pi}(\varphi-\alpha)-\frac{l+p}{2} \frac{i}{\pi} \mid \frac{i}{\pi}\right) \theta_{3}\left(\frac{1}{2 \pi}(\alpha-\psi)-\frac{h+p}{2} \frac{i}{\pi} \mid \frac{i}{\pi}\right), \\
& \theta_{2}\left(\frac{1}{2 \pi}(\varphi-\psi)-\frac{l+h}{2} \frac{i}{\pi} \mid \frac{i}{\pi}\right) \\
& \quad=\frac{1}{2 \pi^{\frac{3}{2}}} \int_{0}^{2 \pi} d \alpha \int_{-\infty}^{\infty} d p e^{-p^{2}} \theta_{2}\left(\frac{1}{2 \pi}(\varphi-\alpha)-\frac{l+p}{2} \frac{i}{\pi} \mid \frac{i}{\pi}\right) \theta_{2}\left(\frac{1}{2 \pi}(\alpha-\psi)-\frac{h+p}{2} \frac{i}{\pi} \mid \frac{i}{\pi}\right) .
\end{aligned}
$$

The authors have not found integral identities for the theta-functions like (4.26) and (4.27) in the literature.

\subsection{The action of the operators}

Finally, we study the action of operators in the Bargmann representation. Let $A$ be a linear operator. Making use of (4.2) we get

$$
(A \phi)\left(\eta^{*}\right)=\frac{1}{4 i \pi^{\frac{3}{2}}} \int_{\mathbf{C}} d \xi d \xi^{*} \frac{e^{-(\ln |\xi|)^{2}}}{|\xi|^{2}} \mathcal{A}\left(\eta^{*}, \xi\right) \phi\left(\xi^{*}\right) .
$$

Equation (4.28) implies that an arbitrary linear operator $A$ is represented in the Bargmann representation by the integral operator. Taking into account (2.14) and (3.10) we find that the kernel $\mathcal{A}\left(\eta^{*}, \xi\right)$ of the integral operator (4.28) can be written as

$$
\mathcal{A}\left(\eta^{*}, \xi\right)=\sum_{j, k=-\infty}^{\infty} A_{j k} \eta^{*-j} \xi^{-k} e^{-\frac{1}{2}\left(j^{2}+k^{2}\right)},
$$

where $A_{j k}=\langle j|A| k\rangle$. On the other hand, we have

$$
\mathcal{A}\left(\eta^{*}, \xi\right)=\langle\eta|A| \xi\rangle
$$

Therefore, the kernel $\mathcal{A}$ is linked to the covariant symbol

$$
A\left(\xi^{*}, \xi\right)=\frac{\langle\xi|A| \xi\rangle}{\langle\xi \mid \xi\rangle}
$$

of the operator $A$ by

$$
\begin{aligned}
\mathcal{A}\left(\xi^{*}, \xi\right) & =\langle\xi \mid \xi\rangle A\left(\xi^{*}, \xi\right) \\
& = \begin{cases}\theta_{3}\left(\frac{i}{\pi} \ln |\xi| \mid \frac{i}{\pi}\right) A\left(\xi^{*}, \xi\right), & \text { (boson case) } \\
\theta_{2}\left(\frac{i}{\pi} \ln |\xi| \mid \frac{i}{\pi}\right) A\left(\xi^{*}, \xi\right) . & \text { (fermion case) }\end{cases}
\end{aligned}
$$

Consider now the linear operators $K_{0}$ and $K_{\frac{1}{2}}$ defined as

$$
\left(K_{0, \frac{1}{2}} \phi\right)\left(\eta^{*}\right):=\frac{1}{4 i \pi^{\frac{3}{2}}} \int_{\mathbf{C}} d \xi d \xi^{*} \frac{e^{-(\ln |\xi|)^{2}}}{|\xi|^{2}} \mathcal{K}_{0, \frac{1}{2}}\left(\eta^{*}, \xi\right) \phi\left(\xi^{*}\right)
$$


where $\mathcal{K}_{0}$ and $\mathcal{K}_{\frac{1}{2}}$ are the reproducing kernels given by (4.25). Evidently, the operators $K_{0}$ and $K_{\frac{1}{2}}$ are the projectors on the space of the irreducible representation of the algebra (2.7), (2.10) and (2.11) corresponding to the case with $j_{0}=0$ (bosons) and $j_{0}=\frac{1}{2}$ (fermions), respectively. Therefore, we can formalize the quantization procedure by demanding that

$$
\left(K_{0} \phi\right)\left(\xi^{*}\right)=\phi\left(\xi^{*}\right), \quad \text { (boson case) }
$$

in the boson case, and

$$
\left(K_{\frac{1}{2}} \phi\right)\left(\xi^{*}\right)=\phi\left(\xi^{*}\right), \quad \text { (fermion case) }
$$

in the case with fermions. Notice that the resolution of the identity (4.2) is rather formal. Actually, we have

$$
\int_{[0,1]}^{\oplus} d j_{0} K_{j_{0}}=I
$$

where $\int^{\oplus}$ designates the direct integral.

\section{Example: free motion in the circle}

We now illustrate the introduced formalism by an example of the Schrödinger equation for the free particle on the circle. We restrict for brevity to the case with bosons. The corresponding Hamiltonian is

$$
\hat{H}=\frac{1}{2} \hat{J}^{2}
$$

By virtue of (2.8) the normalized solution of the Schrödinger equation

$$
\hat{H}|E\rangle=E|E\rangle,
$$

is given by

$$
|E\rangle=|j\rangle, \quad E=\frac{1}{2} j^{2},
$$

where $j$ is integer, that is $\hat{H}$ has purely discrete simple spectrum. It must be borne in mind that due to the twofold degeneracy the vector $|-j\rangle$ is also the normalized solution to (5.2). Clearly, both the solutions are related by (2.12). The fact that the operator of the time inversion $T$ maps the solution $|j\rangle$ into another solution $|-j\rangle$ of the Schrödinger equation (5.2) is a direct consequence of the $T$-invariance of the Hamiltonian (5.1) (see (2.10)). Notice that (3.10) implies the following form of the solution (5.3) in the Bargmann representation:

$$
\phi_{j}\left(\xi^{*}\right) \equiv\langle\xi \mid E\rangle=e^{-\frac{j^{2}}{2}} \xi^{*-j} .
$$


We now discuss the distribution of energies in the coherent state. Taking into account (3.10), (3.28a) and (3.12) we find

$$
\frac{|\langle j \mid \xi\rangle|^{2}}{\langle\xi \mid \xi\rangle}=\frac{|\xi|^{-2 j} e^{-j^{2}}}{\theta_{3}\left(\frac{i}{\pi} \ln |\xi| \mid \frac{i}{\pi}\right)}=\frac{e^{2 l j} e^{-j^{2}}}{\theta_{3}\left(\frac{i l}{\pi} \mid \frac{i}{\pi}\right)} .
$$

Referring back to eq. (3.31) and making use of the definition of the theta-function $\theta_{3}$ (3.26a) we arrive at the approximate relation

$$
\theta_{3}\left(\frac{i l}{\pi} \mid \frac{i}{\pi}\right)=\sqrt{\pi} e^{l^{2}}\left(1+2 \sum_{n=1}^{\infty} e^{-\pi^{2} n^{2}} \cos (2 \pi l n)\right) \approx \sqrt{\pi} e^{l^{2}} .
$$

Using this we can write (5.5) in the following form:

$$
\frac{|\langle j \mid \xi\rangle|^{2}}{\langle\xi \mid \xi\rangle} \approx \frac{1}{\sqrt{\pi}} e^{-(j-l)^{2}}
$$

We have thus shown that the probability of finding the system in the state $|j\rangle$ when the system is in the normalized coherent state $|\xi\rangle / \sqrt{\langle\xi \mid \xi\rangle}$ has a "discrete" Gaussian distribution. Recall that the counterpart of (5.7) in the case of the harmonic oscillator is the asymmetric Poisson distribution.

\section{Conclusion}

We have introduced in this work the coherent states for the quantum particle on the circle. An advantage of the actual approach is that it provides a concrete representation of the algebra (2.7) which seems to be the most natural one for description of the circular motion of a quantum particle. Rather unexpected result is the appearance of the deformed Heisenberg algebra (4.21) in the introduced formalism. There are some indications that this observation would provide some insight into the physical interpretation of quantum deformations. Namely, it seems that the deformed algebra (4.21) would be connected with the lattice-like structure on the classical phase space (the cylinder) implied by the exact relations (3.36) and (3.46) for $l$ integer or halfinteger. This idea is strongly supported by the recent observations of Celeghini et al [18] who demonstrated that in the Fock-Bargmann representation of the quantum WeylHeisenberg algebra in the space of analytic (entire) functions, the coherent and squeezed states and the quantum algebra itself are naturally related to discretized (periodic) quantum systems with the lattice spacing associated with the deformation parameter. Last but not least, we point out that results of this paper would be of importance in the theory of special functions. Indeed, the integral identities for the theta-functions (4.26) and (4.27) are most probably new. 


\section{Acknowledgments}

This work was supported by KBN grant 2P30221706p01. We would like to thank referees for helpful comments.

\section{Appendix A.}

We append the proof of the following approximate relation:

$$
\frac{\left\langle\xi\left|e^{s \hat{J}}\right| \xi\right\rangle}{\langle\xi \mid \xi\rangle} \approx e^{\frac{1}{4} s^{2}+s l} .
$$

Proof. By (2.8), (3.17), (3.18) and (3.29a) (see also (3.30)) we have

$$
\frac{\left\langle\xi\left|\hat{J}^{k}\right| \xi\right\rangle}{\langle\xi \mid \xi\rangle}=\frac{1}{2^{k}} \frac{1}{\theta_{3}} \frac{d^{k} \theta_{3}}{d l^{k}}
$$

Furthermore, as we have already shown (see (3.30)) and (3.38)) the following approximate formula holds:

$$
\frac{1}{\theta_{3}} \frac{d \theta_{3}}{d l} \approx 2 l
$$

Now, consider the identity

$$
\frac{1}{\theta_{3}} \frac{d^{k+1} \theta_{3}}{d l^{k+1}}=\frac{1}{\theta_{3}^{2}} \frac{d \theta_{3}}{d l} \frac{d^{k} \theta_{3}}{d l^{k}}+\frac{d}{d l}\left(\frac{1}{\theta_{3}} \frac{d^{k} \theta_{3}}{d l^{k}}\right),
$$

where $k=0,1,2, \ldots$ In view of $(\mathrm{A} .3)$ we can write $(\mathrm{A} .4)$ in the form of the recursive relation

$$
f_{k+1}=2 l f_{k}+\frac{d f_{k}}{d l}, \quad f_{0} \equiv 1
$$

where

$$
f_{k}=\frac{1}{\theta_{3}} \frac{d^{k} \theta_{3}}{d l^{k}}, \quad k=0,1,2, \ldots
$$

We note that due to (A.3) the equation (A.5) is valid only approximately. Evidently,

$$
\frac{\left\langle\xi\left|e^{s \hat{J}}\right| \xi\right\rangle}{\langle\xi \mid \xi\rangle}=\sum_{k=0}^{\infty} \frac{1}{2^{k}} \frac{f_{k}}{k !} s^{k}
$$

Let us introduce the function $u(s, l)$ such that

$$
u(s, l)=\frac{\left\langle\xi\left|e^{s \hat{J}}\right| \xi\right\rangle}{\langle\xi \mid \xi\rangle}
$$

From (A.5) and (A.7) it follows that

$$
\frac{\partial u(s, l)}{\partial s}=l u(s, l)+\frac{1}{2} \frac{\partial u}{\partial l}, \quad u(0, l) \equiv 1 .
$$


The solution to (A.9) is

$$
u(s, l)=e^{\frac{1}{4} s^{2}+s l} .
$$

This observation completes the proof. We note that the proof refers to the case of bosons. Nevertheless, it can be easily checked (see (3.42) and (3.48)) that the identity (A.1) holds true for fermions as well.

Example: For an easy illustration of (A.1) consider the identity (3.66)

$$
X^{\dagger} X=e^{-2 \hat{J}-1}
$$

On the one hand, using (3.1) and (3.12) we find

$$
\frac{\left\langle\xi\left|X^{\dagger} X\right| \xi\right\rangle}{\langle\xi \mid \xi\rangle}=e^{-2 l} \text {. }
$$

On the other hand, (A.1) implies

$$
\frac{\left\langle\xi\left|e^{-2 \hat{J}-1}\right| \xi\right\rangle}{\langle\xi \mid \xi\rangle} \approx e^{-2 l}
$$

That is in the discussed case the formula (A.1) provides us with the exact result. 


\section{References}

[1] DeWitt-Morette C, private communication

[2] Ali M K 1995 The double pendulum (unpublished)

[3] Emch G G 1972 Algebraic Methods in Statistical Mechanics and Quantum Field Theory (New York: Wiley)

[4] De Bièvre S and González J A 1993 Semiclassical behaviour of coherent states on the circle, in: Ali S T, Ladanov I M and Odzijewicz A, editors, Quantization and Coherent States Methods in Mathematical Physics, Proceedings of 11th Workshop on Geometrical Methods in Mathematical Physics, Bialystok 1992 (Singapore: World Scientific)

[5] Louisell W H 1963 Phys. Lett. 760 Carruthers P and Nieto M M 1968 Rev. Mod. Phys. 40411 Lynch R 1995 Phys. Rep. 256367

[6] Isham C J 1984 Topological and global aspects of quantum theory, in: DeWitt B S and Stora R, editors, Relativity, Groups and Topology II, pp. 1059-1290, Les Houches, Session XL. 1983, Elsevier

[7] Pradhan T and Khare A V 1973 Am. J. Phys. 4159

[8] Ohnuki Y and Kitakado S 1993 J. Math. Phys. 342827

[9] Perelomov A M 1986 Generalized Coherent States and Their Applications (Berlin: Springer)

[10] Barut A O and Girardello L 1971 Commun. Math. Phys. 2141

[11] Whittaker E T and Watson G N 1963 A Course of Modern Analysis (Cambridge: Cambridge University Press)

[12] Bogoliubov N N, Logunov A A and Todorov I T 1975 Introduction to Axiomatic Quantum Field Theory (New York: Benjamin)

[13] Bargmann V 1961 Commun. Pure Appl. Math. 14187

[14] Louisell W H 1964 Radiation and Noise in Quantum Electronics (New York: McGraw-Hill)

[15] Woronowicz S. L. 1991 Commun. Math. Phys. 136399

Brzezinski T, Dabrowski H and Rembielinski J 1992 J. Math. Phys. 3319

[16] Rideau G 1992 Lett. Math. Phys. 24147

[17] Solomon A I 1994 Phys. Lett. A 19629

Wang J, Chuankui W and Jinyu H 1995 Phys. Lett. A 199137

[18] Celeghini E, De Martino S, De Siena S, Vitiello G and Rasetti M 1993 Mod. Phys. Lett. B 20 1321

Celeghini E, De Martino S, De Siena S, Rasetti M and Vitiello G 1995 Ann. of Phys. (N.Y.)

24150 